\title{
Monocyte metabolic transcriptional programs associate with resistance to tuberculin skin test/interferon- $\gamma$ release assay conversion
}

\author{
Jason D. Simmons, ${ }^{1}$ Phu T. Van, ${ }^{2}$ Catherine M. Stein,, ${ }^{3,4}$ Violet Chihota, ${ }^{5,6}$ Thobani Ntshiqa, ${ }^{6}$ Pholo Maenetje, ${ }^{6}$ Glenna J. Peterson, ${ }^{1}$ \\ Anthony Reynolds, ${ }^{1}$ Penelope Benchek, ${ }^{3}$ Kavindhran Velen, ${ }^{6}$ Katherine L. Fielding, ${ }^{5,7}$ Alison D. Grant,, ${ }^{5,78}$ Andrew D. Graustein, ${ }^{1}$ \\ Felicia K. Nguyen, ${ }^{1}$ Chetan Seshadri, ${ }^{1}$ Raphael Gottardo, ${ }^{2}$ Harriet Mayanja-Kizza, ${ }^{9}$ Robert S. Wallis, ${ }^{6}$ Gavin Churchyard, ${ }^{6}$ \\ W. Henry Boom, ${ }^{4}$ and Thomas R. Hawn ${ }^{1}$ \\ ${ }^{1}$ TB Research and Training Center, Department of Medicine, University of Washington, Seattle, Washington, USA. ${ }^{2}$ Fred Hutchinson Cancer Research Center, Seattle, Washington, USA. ${ }^{3}$ Department of \\ Population \& Quantitative Health Sciences and ${ }^{4}$ Department of Medicine, Case Western Reserve University, Cleveland, Ohio, USA. ${ }^{5}$ School of Public Health, University of Witwatersrand, Johannesburg, \\ South Africa. ${ }^{6}$ The Aurum Institute, Parktown, South Africa. ${ }^{7}$ TB Centre, London School of Hygiene and Tropical Medicine, London, United Kingdom. ${ }^{8}$ Africa Health Research Institute, School of Nursing and \\ Public Health, University of KwaZulu-Natal, Durban, South Africa. ${ }^{9}$ Department of Medicine, School of Medicine, Makerere University, Kampala, Uganda.
}

\begin{abstract}
After extensive exposure to Mycobacterium tuberculosis (Mtb), most individuals acquire latent Mtb infection (LTBI) defined by a positive tuberculin skin test (TST) or interferon- $\gamma$ release assay (IGRA). To identify mechanisms of resistance to Mtb infection, we compared transcriptional profiles from highly exposed contacts who resist TST/ICRA conversion (resisters, RSTRs) and controls with LTBI using RNAseq. Gene sets related to carbon metabolism and free fatty acid (FFA) transcriptional responses enriched across 2 independent cohorts suggesting RSTR and LTBI monocytes have distinct activation states. We compared intracellular Mtb replication in macrophages treated with FFAs and found that palmitic acid (PA), but not oleic acid (OA), enhanced Mtb intracellular growth. This PA activity correlated with its inhibition of proinflammatory cytokines in Mtbinfected cells. Mtb growth restriction in PA-treated macrophages was restored by activation of AMP kinase (AMPK), a central host metabolic regulator known to be inhibited by PA. Finally, we genotyped AMPK variants and found 7 SNPs in PRKAG2, which encodes the AMPK- $\gamma$ subunit, that strongly associated with RSTR status. Taken together, RSTR and LTBI phenotypes are distinguished by FFA transcriptional programs and by genetic variation in a central metabolic regulator, which suggests immunometabolic pathways regulate TST/IGRA conversion.
\end{abstract}

\section{Introduction}

Tuberculosis (TB) was the leading cause of death from a single infectious agent worldwide in 2019, accounting for 1.5 million deaths (1). One contributor to this burden of disease is the large reservoir of asymptomatic Mycobacterium tuberculosis (Mtb) infections that affects approximately a quarter of the world's population (2). Despite this prevalence, some individuals show no evidence of Mtb infection even after heavy and prolonged exposures and may be considered relatively resistant to infection (3). Characterization of this Mtb resistance phenotype is an emerging area of research (4-12) that may uncover novel immunologic pathways amenable to host-directed therapy or vaccine development.

Latent Mtb infection (LTBI) is defined as a positive tuberculin skin test (TST) or interferon- $\gamma($ IFN- $\gamma$ ) release assay (IGRA) in an individual without clinical or radiographic evidence of TB. Individuals who are resistant to LTBI (resisters [RSTRs]) have persistently

Conflict of interest: The authors have declared that no conflict of interest exists. Copyright: ( 2021 , Simmons et al. This is an open access article published under the terms of the Creative Commons Attribution 4.0 International License.

Submitted: May 8, 2020; Accepted: June 3, 2021; Published: June 10, 2021.

Reference information: J Clin Invest. 2021;131(14):e140073.

https://doi.org/10.1172/JCl140073. negative TST/IGRA testing despite intense and prolonged aerosol exposure to Mtb (3). We and others have identified RSTR populations among household TB contacts in Uganda (7), India (5), the Gambia (6), Haiti (12), and Indonesia $(9,10)$. While immunologic profiling of these cohorts suggests differences between RSTR and LTBI phenotypes $(4,6,10,11)$, to date no immunologic RSTR signature emerged across studies, which may be due to heterogeneity in case definitions, genetic background, Mtb exposure, duration of follow-up, and types of immune profiling assays that were employed. To our knowledge, no studies compared responses in a single cell type across multiple, stringently defined RSTR cohorts where conserved pathways are more likely to be identified.

Resident alveolar macrophages and recruited monocytederived macrophages are early targets of Mtb in the lung (13-15) and poised to play central roles in resistance phenotypes such as early innate clearance or the priming of alternative $\mathrm{T}$ cell responses $(3,4)$. In the current study, we aimed to find conserved monocyte immunologic signatures across 2 distinct RSTR cohorts, each of which had lengthy follow-up after high Mtb exposure. In Ugan$\mathrm{da}$, we enrolled household contacts of pulmonary TB cases and followed them for 8 to 10 years with serial TST and IGRA testing $(7,8)$. In South Africa, we enrolled gold miners who had worked $\geq$ 15 years in the mining industry, an environment with documented 
A

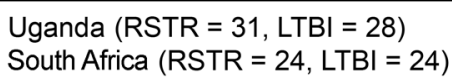

Thaw Paxgene RNA samples

RNA isolation, globin depletion

RNA sequencing

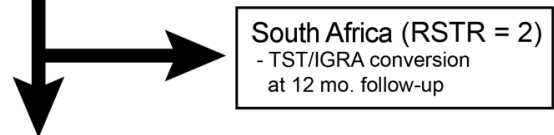

Uganda (RSTR $=31, \mathrm{LTBI}=28)$

South Africa $(R S T R=22, L T B I=24)$

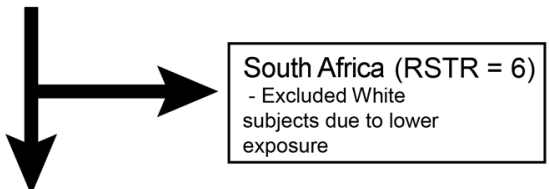

Uganda $(\mathrm{RSTR}=31, \mathrm{LTBI}=28)$

South Africa $(R S T R=16, L T B \mid=24)$

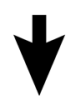

Differential Gene Expression

(RSTR vs. LTBI)
C

Uganda $(\mathrm{RSTR}=43, \mathrm{LTBI}=49)$

South Africa $(R S T R=29$, LTBI $=30)$

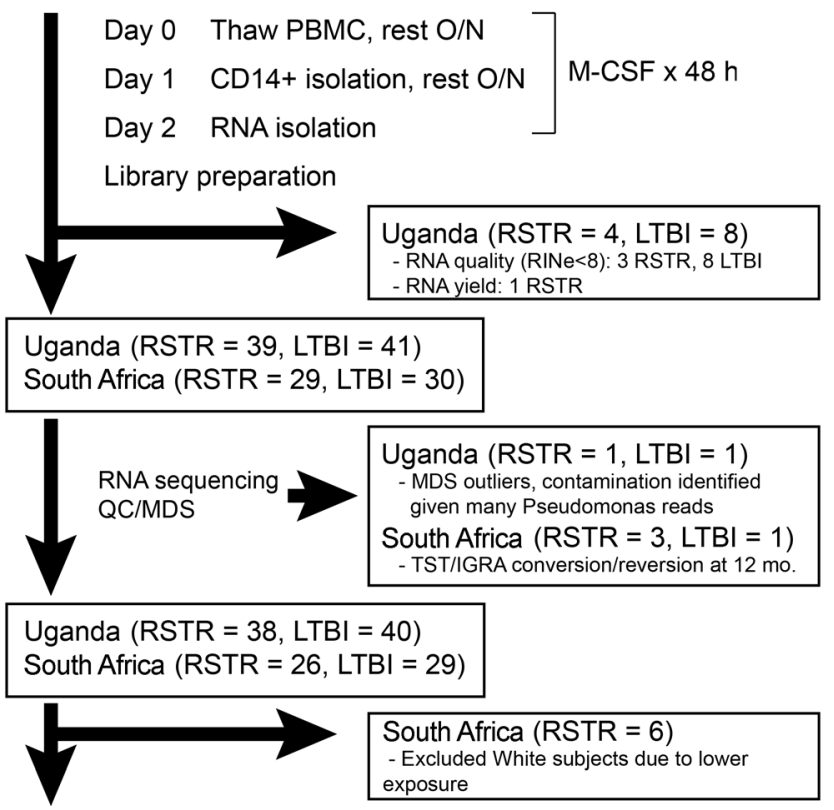

Uganda $(\mathrm{RSTR}=38, \mathrm{LTBI}=40$ )

South Africa $(R S T R=20, L T B I=29)$

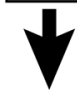

Differential Gene Expression

(RSTR vs. LTBI)
B

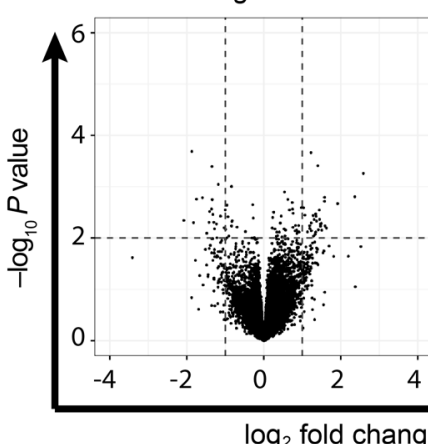

Uganda

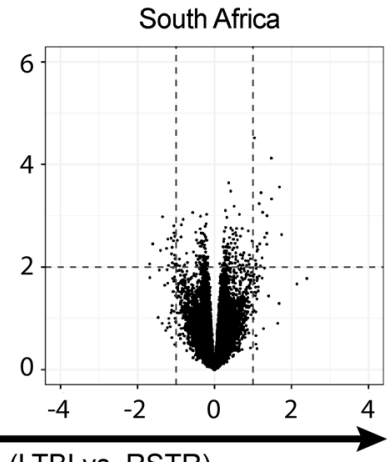

(LTBI vs. RSTR)

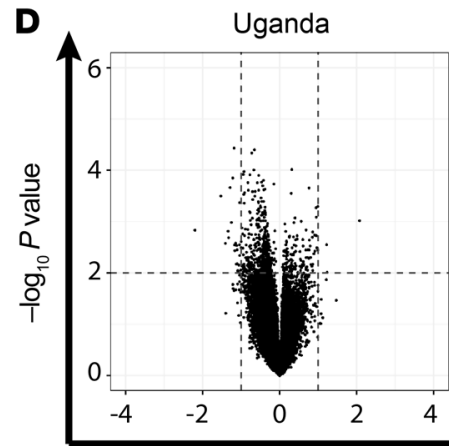

$\log _{2}$ fold change (LTB| vs. RSTR)

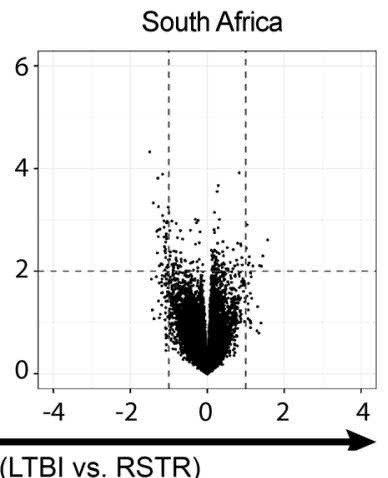

Figure 1. Comparison of RSTR and LTBI gene expression in whole blood and monocytes from Ugandan household Mtb contacts and from gold miners in South Africa. RNA from whole blood of Ugandan and South African donors was analyzed by RNAseq. (A and B) Differential gene expression between
RSTR and LTBI donors were compared after exclusion of the indicated samples. (C and D) CD14 $4^{+}$monocytes were purified from cryopreserved PBMCs and differentiated in M-CSF for 48 hours before RNA isolation and sequencing. In Uganda, 50 subjects were included in both analyses (26 RSTR, 24 LTBI), while additional unique subjects were included in the monocyte (12 RSTR and 16 LTBI) and whole blood (5 RSTR and 4 LTBI) analyses. In South Africa, 39 of 40 subjects included in the whole blood analysis were also included in the monocyte analysis. Volcano plots identify no single genes that were significantly (FDR < 0.2) differentially expressed between RSTR and LTBI subjects in either population in the whole blood (B) and monocyte (D) analyses.

extreme levels of Mtb transmission due to employment conditions in confined spaces, congregate living arrangements, and comorbid conditions including HIV and silicosis, among other factors $(16,17)$. Among these gold miner subjects, where LTBI prevalence has been measured at $89 \%$ (17) with an annual risk of infection estimated to be $10 \%$ to $20 \%$ (18), we defined RSTRs as those with negative TST/IGRA testing.
In these Ugandan and South African cohorts, we identified monocyte transcriptional profiles that distinguish RSTR and LTBI populations and that suggest differences in central carbon metabolism and free fatty acid (FFA) signaling pathways. Furthermore, we identify associations between polymorphisms in a gene encoding the metabolic regulator AMPK and the RSTR phenotype. Metabolic shifts are increasingly recognized as relevant to both Mtb 
A Enrichment of canonical pathways, chemical perturbation, immunologic signatures (MsigDB collection $\mathrm{C} 2+\mathrm{C} 7$ ) FDR $<0.01$

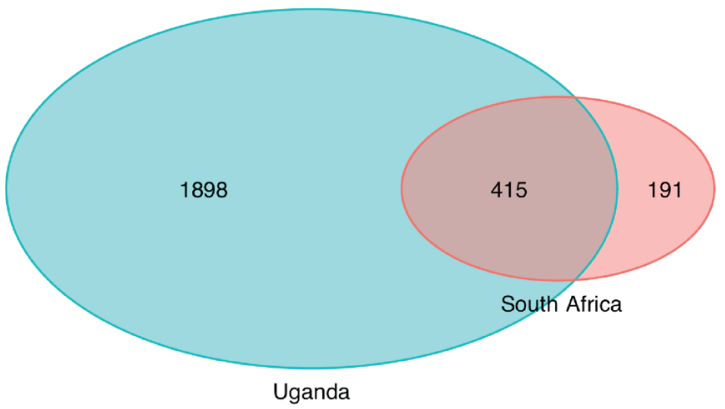

Overlapping

Gene Set Category $(\mathrm{C} 2+\mathrm{C} 7)$

Central carbon metabolism

Antiviral/Type 1 IFN

Contrasting cell populations

Other

Total
B

\section{Enrichment of Gene Ontology terms \\ (MSigDB collection C5) FDR $<0.01$}

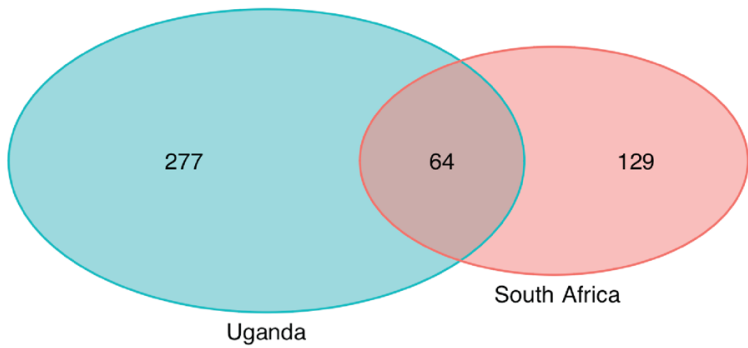

Gene Set Category (C5)
Central carbon metabolism
Nucleoside metabolism
RNA metabolism
Ribosome/protein synthesis
Other

Overlapping

Gene sets

25

6

3

10

20

Total
64

Figure 2. Differentially expressed gene sets in RSTR versus LTBI donors are shared across the Ugandan and South African cohorts. (A) Using the CAMERA gene set test (30) on curated gene sets available from the Molecular Signatures Database (MSigDB, C2 and C7), many transcriptional programs were enriched (FDR < 0.01) with RSTR or LTBI phenotypes in Uganda ( $n=2313$ gene sets) and in South Africa ( $n=606$ gene sets). Among the gene sets with enrichment in both populations $(n=415)$, type 1 interferon-driven transcriptional responses enriched among RSTR and LTBI donors whereas genes involved in metabolic programs (e.g., oxidative phosphorylation, TCA cycle) uniformly had higher expression among RSTR donors. (B) Similar enrichment of metabolic programs among RSTR donors across cohorts was also identified when gene sets curated by GO terms were analyzed by CAMERA (MSigDB, C5).

virulence and the host response (19-24); our findings suggest that RSTRs have differential fatty acid responses that may impact basal metabolic activities to restrict TST/IGRA conversion.

\section{Results}

RSTR and LTBI subjects have similar blood transcriptional profiles and cellularity. Blood transcriptional profiles have successfully distinguished asymptomatic Mtb infection from Mtb disease (25-28). We asked whether blood transcriptomes could distinguish RSTR from LTBI phenotypes among TB household contacts in Uganda and among gold miners in South Africa. Using RNA sequencing, we compared whole blood transcriptomes from donors in Uganda (31 RSTR versus 28 LTBI subjects) and South Africa (22 RSTR versus 24 LTBI subjects; Figure 1A). Demographic and epidemiologic characteristics between RSTR and LTBI were similar in Uganda including the Mtb exposure score (Supplemental Table 1; supplemental material available online with this article; https://doi.org/10.1172/ JCI140073DS1), whereas in South Africa (Supplemental Table 2), ethnicity significantly associated with RSTR status $(P=0.003)$. We stratified subsequent South Africa analyses according to the dominant ethnic group. After adjusting for multiple comparisons, no genes were significantly differentially expressed between RSTR and LTBI phenotypes in either population (false discovery rate [FDR] $<0.2$; Figure 1B and Supplemental Tables 3 and 4). Using immunoStates deconvolution (29), we found minimal, if any, differences in the computationally predicted frequencies of cellular populations between RSTR and LTBI donor blood (Supplemental Figures 1 and 2). Furthermore, we found no difference in the fre- quency of $\mathrm{CD} 4^{+}$cells, T cell subsets, NK cells, or B cells in PBMCs isolated from Ugandan donors when measured by flow cytometry (23 RSTR versus 23 LTBI subjects, Supplemental Figure 3). In summary, RSTR and LTBI subjects showed no differences in global gene transcription or frequency of cellular subsets in whole blood.

Metabolic gene programs are enriched among RSTR monocytes across cohorts. We next hypothesized that monocytes regulate TST/ IGRA conversion due to their potential to regulate early Mtb clearance or T cell priming in highly exposed subjects. CD14 ${ }^{+}$monocytes were isolated from PBMCs obtained from RSTR (Uganda $n=$ 38; South Africa $n=26$ ) and LTBI (Uganda $n=40$; South Africa $n=$ 29) subjects from which RNA was isolated and examined by RNA sequencing (Figure 1C). Both demographic characteristics and exposure scores were similar between RSTR and LTBI donors in Uganda (Supplemental Table 5) whereas in South Africa, ethnicity associated with RSTR status ( $P=0.003$, Supplemental Table 6). Accordingly, we performed all analyses stratified by ethnicity and present results according to the dominant ethnic group (Figure 1C). Using multidimensional scaling (MDS), we found no evidence of batch effects nor any global influence of age or other available metadata when plotting the first 2 dimensions (Supplemental Figures 4 and 5). We found no differentially expressed genes (DEGs) in either cohort after multiple testing correction (FDR $<0.2$; Figure 1D and Supplemental Tables 7 and 8).

We next examined whether additive biologic effects of gene networks differed in the LTBI or RSTR transcriptomes using a competitive gene set test, CAMERA (30), which is analogous to gene set enrichment analysis (GSEA) (31). Using approximately 
A

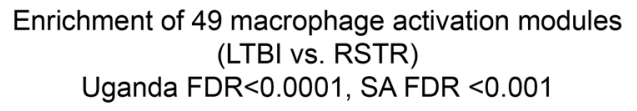

Enrichment of 49 macrophage activation modules (LTBI vS. RSTR)

Uganda FDR $<0.0001$, SA FDR $<0.001$

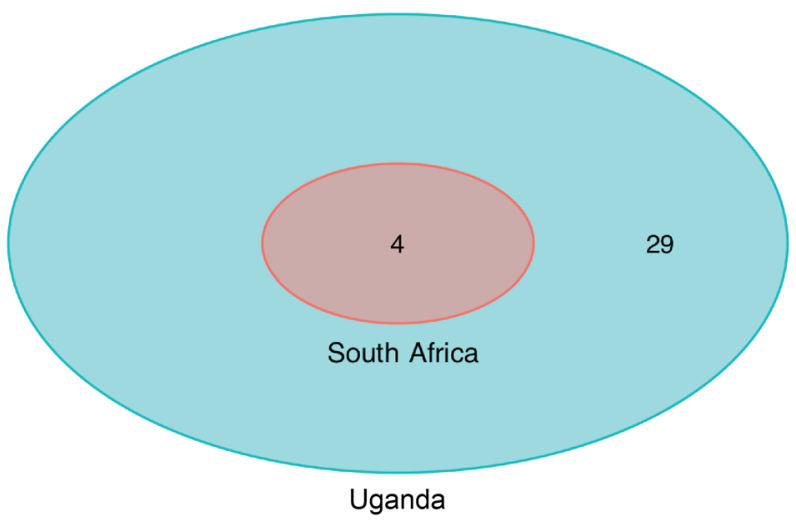

B

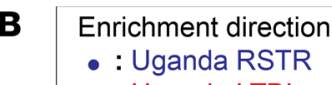

- : Uganda LTBI

Module \# ['06']: South Africa RSTR

Module \# ['22']: South Africa LTBI

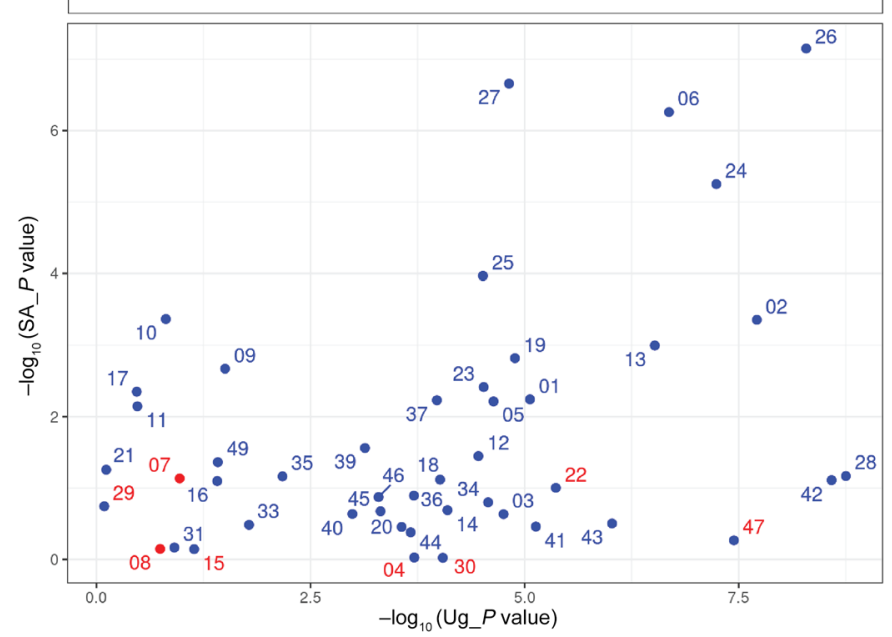

Figure 3. Gene sets derived from FFA-stimulated macrophages are consistently enriched among RSTR donors across the Ugandan and South African cohorts. Gene sets related to a variety of macrophage stimuli were obtained from previously published weighted gene coexpression analysis (WCCNA) modules (33). Enrichment among RSTR vs. LTBI transcriptomes in any of these 49 WGCNA modules was analyzed using CAMERA gene set enrichment (30). (A) All modules with significant enrichment in South Africa were also enriched in Uganda (FDR $<0.001)$. (B) The correlation plot compares $P$ values from CAMERA enrichment scores in Uganda ( $x$ axis) versus South Africa ( $y$ axis) and indicates the corresponding direction of enrichment with RSTR phenotype (red) or LTBI phenotype (blue). This enrichment direction among Ugandan monocyte donors (color of data point) and among South African monocyte donors (color of module number) is highly concordant. Gene sets that correlate to macrophage FFA stimulations (Set 27, Set 6, Set 24, Set 26) were enriched among RSTR donors in Uganda and South Africa.

10,000 curated gene sets from the Molecular Signatures Database (MSigDB) $(31,32)$, we identified 2313 gene sets in monocytes from Ugandan participants and 606 gene sets in monocytes from South African participants, with enrichment for either the RSTR or LTBI phenotypes (FDR < 0.01). Strikingly, 415 of these enriched gene sets were common across populations (Figure 2A and Supplemental Table 9). Importantly, the direction of enrichment of these overlapping gene sets was consistent across both populations. Several cellular pathways associated with each clinical phenotype, including the IFN $-\alpha / \beta$ response ( $n=31$ gene sets) and mitochondrial respiration or carbon metabolism ( $n=32$ gene sets). Genes involved in the TCA cycle or oxidative phosphorylation uniformly had higher expression in RSTR as compared with LTBI monocytes (Supplemental Table 9, direction 'UP'). Finally, using gene sets derived from gene ontology (GO) terms (MSigDB C5 collection), CAMERA gene set enrichment identified a total of 64 overlapping biologic pathways (FDR < 0.01; Figure 2B and Supplemental Table 10). Many of these GO terms (25 of 64) related to mitochondrial respiration or oxidoreductase activities in addition to other metabolic processes. Overall, these results identify conserved transcriptional programs that underly Mtb resistance in the Ugandan and South African cohorts and suggest distinct metabolic activities or activation states.

Gene set enrichment suggests distinct fatty acid activation states in RSTR monocytes. To better define macrophage activation states that distinguish RSTR monocytes, we evaluated whether transcriptional modules related to a panel of macrophage stimuli are enriched among RSTR versus LTBI monocytes. We examined 49 gene sets derived from WGCNA transcriptional modules identified by Xue et al. (33) who stimulated MDMs with diverse activation signals including type I and type II IFN, proinflammatory and antiinflammatory cytokines, glucocorticoids, TLR ligands, lipoproteins, and FFAs. Using CAMERA to analyze these 49 macrophage activation modules, we found significant enrichment of multiple modules with the RSTR phenotype (Supplemental Tables 11 and 12). Among 33 macrophage stimulation modules that were enriched in Uganda (FDR $<0.0001)$, 4 were also enriched in South Africa (FDR < 0.001; Figure 3A). These 4 overlapping modules included genes with higher expression among RSTR (as compared with LTBI) monocytes (Sets 27, 26, 06, and 24; Figure 3B).

To identify the cellular processes represented by these 4 overlapping transcriptional modules, we next evaluated which macrophage stimuli most significantly contributed to each module. Correlation between each module eigengene and each original macrophage stimulus reported by Xue et al. (33) are depicted in Supplemental Figure 6. Clustering of these stimuli suggested that the expression pattern in resting monocytes from RSTRs was most influenced by the cellular responses to FFAs. Specifically, several modules had strong, positive correlation with long-chain FFA oleic acid (OA) and linoleic acid (LiA) (Sets 6, 24, and 26) or with lauric acid (LA) and high-density lipoprotein (HDL) (Set 27). Genes within these modules had higher expression among RSTR resting monocytes, which indicates that metabolic pathways influenced by OA/LiA (and LA/HDL) may play a role in the resistance phenotype. Taken together, these data suggest differential metabolic activities between RSTR and LTBI monocytes that relate to the cellular response to FFAs. 
A

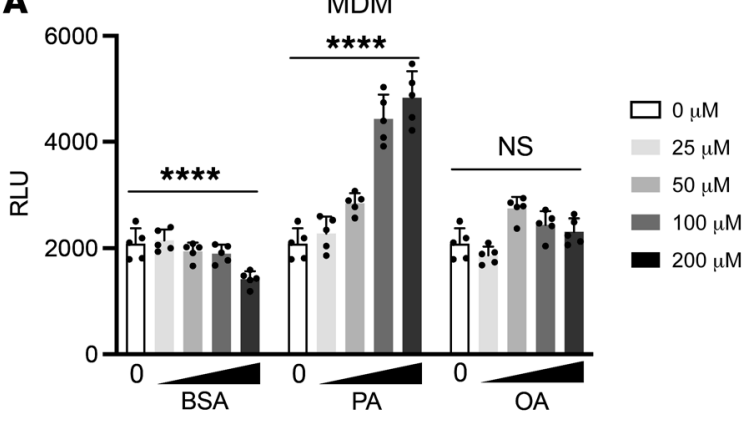

B

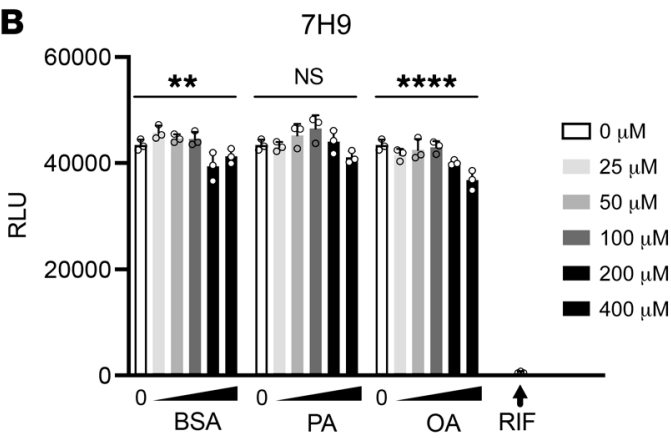

Figure 4. Control of intracellular Mtb growth is lost in PA-treated macrophages. (A) Human MDMs were infected with a reporter Mtb Erdman strain (Mtb-lux) for 4 hours $(\mathrm{MOI}=2)$, washed, then treated with $\mathrm{PA}, \mathrm{OA}$, or vehicle controls that were matched for BSA content. (B) Mtb-lux was diluted in complete $7 \mathrm{H} 9$ media containing indicated concentrations of $\mathrm{OA}$, PA, matched BSA controls, or $0.5 \mu \mathrm{g} / \mathrm{mL}$ rifampin (RIF) to an $\mathrm{OD}$ approximately 0.005 . Replication was measured as RLUs after 3 days in each experiment. Means are plotted for biologic replicates (A, $n=5$ replicates; $\mathbf{B}, n=3$ replicates) along with standard deviation (error bar). Effects are representative of more than 5 independent experiments using a total of 2 donors (A, macrophages), or of 2 independent experiments (B); a simple linear model was applied to test for significant dose-dependent effects on Mtb growth. NS, $P>0.05 ;{ }^{* *} P<0.01 ;{ }^{* * *} P<0.0001$.

$P A$, but not OA, potentiates intracellular Mtb growth and modulates macrophage cytokine responses. OA and PA are the 2 the most abundant circulating FFAs in humans and have distinct, or even antagonistic, immunomodulatory properties (34). Interestingly, the only Xue et al. (33) gene module with significant enrichment (FDR < 0.15) among LTBI monocytes across the Ugandan and South African cohorts (Set 7; Figure 3B) consists of a macrophage gene expression profile that correlates with PA and IFN- $\gamma$ stimulation. We next hypothesized that the early cellular Mtb response would differ in OA- versus PA-treated macrophages, considering that the transcriptional patterns following stimulation with these FFA species overlaps with resting RSTR versus LTBI monocytes, respectively. Mtb-infected healthy-donor MDMs were treated with OA and PA complexed to bovine serum albumin (BSA), which is required for solubilization. Intracellular Mtb growth was potentiated by PA in a dose-dependent manner, whereas OA had minimal effect (Figure 4A). PA and OA were minimally toxic to macrophages at these doses (Supplemental Figure 7A). PA was toxic at doses greater than or equal to $400 \mu \mathrm{M}$ (36\% cell death) yet further potentiated intracellular Mtb replication despite this reduced macrophage viability (Supplemental Figure 7B). Both PA and OA are mycobacterial carbon sources yet have also been associated with direct microbicidal activity $(35,36)$. In 7 H9 broth, neither PA nor OA had pronounced effects on Mtb growth (Figure 4B), suggesting that PA most likely modulates macrophage function or perhaps influences Mtb nutrient acquisition within the macrophage environment.

To explore the mechanisms by which PA enhances Mtb growth within macrophages, we first compared effects of OA and PA on macrophage cytokine production in response to antigens present in Mtb whole cell lysate (TBWCL). Both OA and PA potentiated TNF secretion and inhibited IL-10 secretion in a dose-dependent manner, whereas only PA robustly potentiated IL-1 $\beta$ secretion at doses greater than or equal to $200 \mu \mathrm{M}$ (Figure 5A). In contrast, proinflammatory cytokine secretion by Mtb-infected macrophages was inhibited by PA (Figure 5B) while OA had no effect. The inhibitory effect on proinflammatory cytokine secretion from Mtb-infected macrophages suggests a possible mechanism whereby PA limits the macrophage capacity to restrict Mtb growth.
In addition to effects on proinflammatory responses, PA modulates multiple macrophage pathways that include the inhibition of the AMPK (37), a central energy-sensing serine/ threonine kinase that promotes catabolic processes in response to low AMP/ATP ratios. To evaluate whether rescuing AMPK activity would restore control of intracellular Mtb growth in PA-treated macrophages, we treated Mtb-infected MDMs with PA $(100 \mu \mathrm{M})$ in the presence or absence of a potent AMPK activator (A 769662). At nontoxic A 769662 doses (100-200 $\mu \mathrm{M})$, AMPK activation limited Mtb growth in a dose-dependent manner, restoring growth restriction to levels observed in cells not treated with PA (Figure 6A). PA-mediated inhibition of AMPK results in potentiation of LPS-induced NLRP3 inflammasome activation (37). However, in the context of Mtb-infected cells, PA had inhibitory effects on IL-1 $\beta$ secretion (Figure $5 \mathrm{~B}$ ), and control of intracellular Mtb growth in U937 cells was independent of NLRP3 (and NLRC4; Figure 6B). These data suggest that PA targets pathways independent of AMPK-mediated NLRP3 activation. AMPK also regulates autophagy by directly phosphorylating ULK1, which catalyzes an initiation complex for LC3 conjugation and autophagosome formation, activities that are inhibited by PA (37). However, the PA effects on intracellular Mtb growth restriction were independent of ULK1 in U937 cells (Figure 6B). In summary, PA disrupts protective macrophage proinflammatory cytokine responses and results in a reduced capacity to restrict Mtb growth, which can be restored through AMPK activation.

PRKAG2 polymorphisms are associated with resistance to TST/ IGRA conversion. Since FFA transcriptional signatures distinguished RSTR and LTBI monocytes, and AMPK activity modulated FFA effects on intracellular Mtb growth, we next explored a direct link between AMPK and the Mtb resistance phenotype. We used a genetic approach to examine whether polymorphisms in AMPK subunit genes are associated with resistance to TST/IGRA conversion. This complex is comprised of a catalytic $\alpha$-subunit and regulatory $\beta$ - and $\gamma$-subunits, each of which has multiple isoforms (38). We examined 257 SNPs within the 7 genes that encode the $\alpha$-subunit isoforms (PRKAA1 and A2), the $\beta$-subunit isoforms (PRKAB1 and B2), and the $\gamma$-subunit isoforms (PRKAG1, G2 and G3) for associations with RSTR 
A
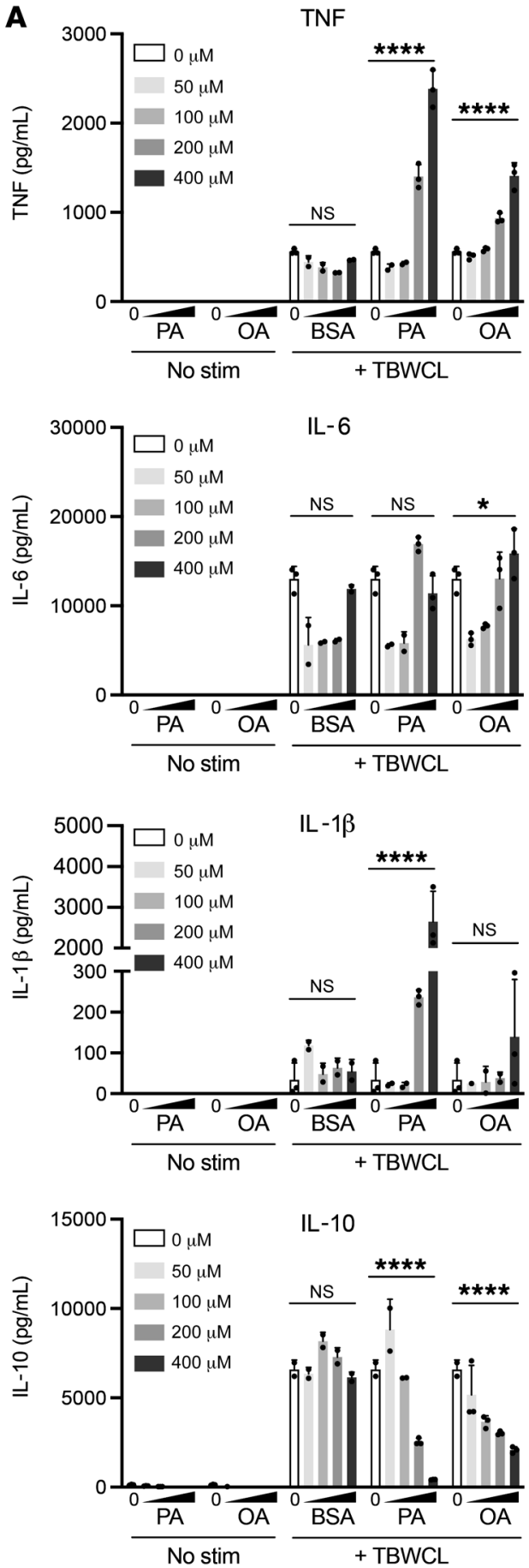

B
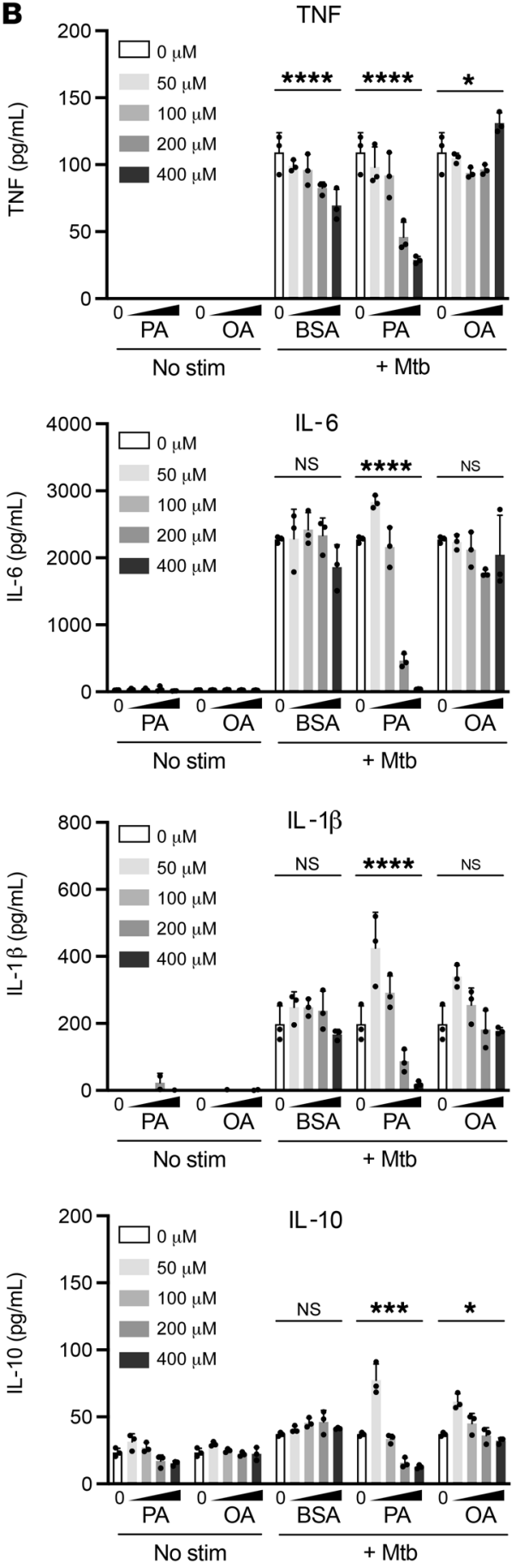

Figure 5. OA and PA modulate macrophage cytokine responses, but only PA restricts proinflammatory cytokines following Mtb infection. Healthy human MDMs were treated with the indicated doses of $\mathrm{PA}, \mathrm{OA}$, or controls matched for BSA content overnight and then stimulated with (A) Mtb whole cell lysate (TBWCL), (B) infected with live Mtb (5 CFU per cell), or left unstimulated. Cytokine secretion was quantitated by ELISA from supernatants at 24 hours after stimulation. Means are plotted for biologic replicates (A, $n=2$ or 3 replicates; $\mathbf{B}, n=3$ replicates) along with standard deviation (error bar). Effects are representative of 2 independent experiments from a single donor. Data were fit using a simple linear model to test for a significant dose-dependent effect and were log-transformed as necessary to achieve the best regression fit. NS, $P>0.05$; ${ }^{*} P<0.05$; ${ }^{* *} P<0.001$; ****P $<0.0001$.
( $n=74$ genotyped subjects) or LTBI ( $n=189$ genotyped subjects) phenotypes among Ugandan household contacts (7). Seven SNPs within PRKAG2 were strongly associated with RSTR status (OR 1.9-3.2, P $<0.005$; Table 1) using an additive genetic model adjusted for kinship, sex, and age. Inheritance of 2 copies of the minor allele further increased the association with RSTR status with odds ratios ranging from 3.3 to 18.5 (Supplemental Table 13, recessive model). When corrected for false discovery, 3 of the 7 SNPs remained significant (FDR < 0.05; rs10480299, rs10480300, rs114166988), all of which are in moderate linkage disequilibrium, suggesting a single causative locus (Supplemental Table 14). These results suggest that genetic variation in a central regulator of metabolism is associated with the RSTR outcome and is highly consistent with our ex vivo monocyte transcriptomic profiling findings. Moreover, the previously described inhibition of AMPK by PA (37), which we demonstrate enhances intracellular Mtb growth, further implicates differential AMPK activity in monocyte responses between RSTR and LTBI contacts.

\section{Discussion}

Protective mechanisms that restrict TST/IGRA conversion in highly exposed contacts are not defined. Our study suggests that RSTR and LTBI populations have distinct monocyte transcriptional pro- 
A

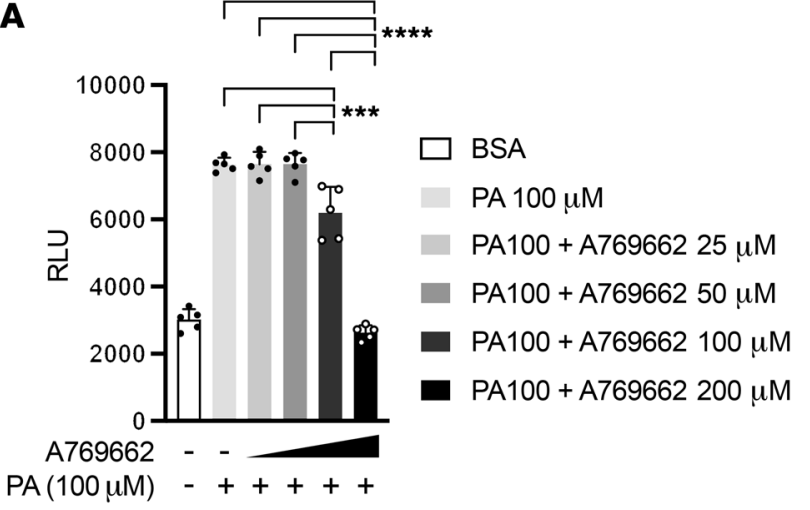

Figure 6. Control of intracellular Mtb growth in PA-treated macrophages is restored by AMPK activation. (A) Healthy human donor MDMs were infected with a reporter Mtb Erdman strain (Mtb-lux) for 4 hours ( $\mathrm{MOI}=2$ ), washed, then treated with $100 \mu \mathrm{M}$ PA alone or in combination with the indicated doses of the AMPK activator A 769669. (B) U937 monocytes with disruptions in inflammasome genes (encoding NLRP3 or NLRC4), an autophagy gene (ULK1), or control cell lines expressing nontargeting guide RNAs were generated by CRISPR-Cas 9 technology, differentiated in PMA and infected with Mtb-lux as in (A), then treated with 200 $\mu \mathrm{M}$ PA or BSA vehicle control. RLUs were recorded at 3 days after infection ( $\mathbf{A}$ and B); mean RLU of 5 biologic replicates are plotted with standard deviations (error bars). Findings are representative of 2 independent experiments (A, B) from a single donor (A). The effect of AMPK activation on Mtb-lux growth in PA-treated cells was significant as determined by $(\mathbf{A}) 1$-way ANOVA $(P<0.0001)$, where $P$ values were then calculated for the indicated pairwise comparisons and adjusted using Tukey's multiple comparison test. $P$ values for single-dose PA effects were calculated by 2 -tailed $t$ test $(\mathbf{B}) .{ }^{* * *} P<0.001$; ${ }^{* * *} P<0.0001$.
B

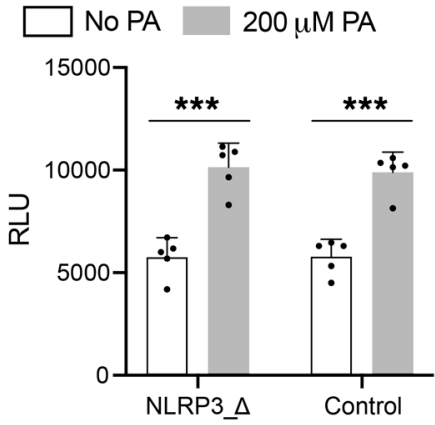

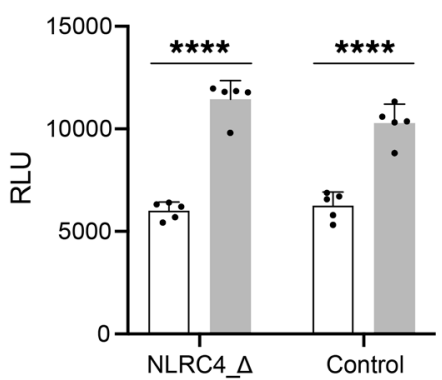

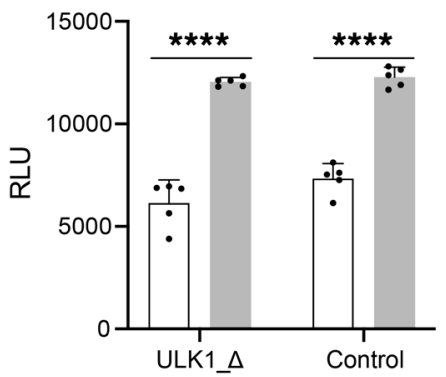

files that relate to differences in metabolic pathways and the cellular response to FFAs. Transcriptional profiles of OA-conditioned macrophages were enriched in RSTR as compared with LTBI monocytes. Consistent with their known opposing activities on inflammatory pathways, we found that PA and not OA enhanced Mtb growth within macrophages that may relate to distinct intracellular Mtb control pathways in RSTR and LTBI individuals. In mechanistic support of these exploratory findings, we identify AMPK polymorphisms that strongly associate with the RSTR phenotype and found that pharmacologic restoration of AMPK function abrogates PA-mediated cellular effects on Mtb growth in vitro. Taken together, our study suggests that RSTR monocytes have distinct metabolic activities that mediate resistance to TST/ IGRA conversion and identify AMPK as a potential regulator.

Immunometabolic pathways are central to host-Mtb interactions and regulate infection outcomes $(24,39-43)$. Several reports indicate that Mtb induces macrophage glycolysis to its benefit (24, 44 ), whereas macrophage subtypes that are skewed toward glycolytic metabolism are less permissive to Mtb in vivo (22). The shift away from oxidative phosphorylation and toward aerobic glycolysis is a hallmark of macrophage activation by TLR ligands and IFN- $\gamma$ $(45,46)$, and is linked to host-protective mechanisms regulated by HIF-1 $\alpha(21,42,47)$. Our gene set enrichment analyses identified multiple gene sets linked to mitochondrial respiration and the TCA cycle and these genes collectively had higher expression among resting RSTR relative to LTBI monocytes. Interestingly, a whole blood transcriptional signature that most strongly predicted treatment failure among subjects with Mtb disease included suppressed mitochondrial genes prior to treatment initiation (48), which suggests that adequate expression levels may reflect a host response that not only correlates with resistance to infection but also respon- siveness to treatment. Whether the association of oxidative phosphorylation gene sets among RSTR cells translates to a differential flux through the mitochondrial electron transport chain, or if such perturbations are also differentially regulated in RSTR versus LTBI monocytes after infection with Mtb, is a focus of future studies.

Our second major finding genetically links the RSTR phenotype to a central metabolic regulator. This locus (chromosome 7q36.1), encoding the AMPK- $\gamma$ subunit, has not previously been associated with the RSTR phenotype. However, these PRKAG2 SNP associations are of a similar confidence $(P=0.0003-$ $0.00001)$ as previously identified genetic loci that associate with resistance to TST conversion, accounting for differences in study design (49-53). Additional studies are required to determine the functional PRKAG2 SNPs, whether they increase or decrease AMPK activity, and characterize the downstream immunometabolic effects that may contribute to resistance to Mtb infection. Of interest, multiple host-directed therapies that enhance macrophage restriction of Mtb intracellular growth are known to positively regulate activity of AMPK-linked pathways including metformin (43), sirtuin-1 activators $(19,54)$, and AICAR (55). We also found AMPK activation with A 769662 restricted Mtb growth in macrophages (Figure 5A). Notably, metformin use (and by extension perhaps AMPK activation) has also been associated with reduced prevalence of LTBI, which suggests activity that restricts incident infection in vivo $(56,57)$. Additional studies are required to directly determine whether AMPK activity differs in primary cells isolated from RSTR and LTBI subjects.

We also found that gene sets related to FFA stimulation were differentially enriched in RSTR versus LTBI monocytes under resting conditions. Interestingly, metabolomic signatures of medium-chain and long-chain fatty acid plasma concentrations were 


\section{Table 1. Polymorphisms in PRKAG2, which encodes a regulatory AMPK subunit, are associated with the RSTR phenotype in Uganda}

\begin{tabular}{|c|c|c|c|c|c|c|}
\hline \multirow[b]{2}{*}{ SNP } & \multicolumn{4}{|c|}{ Genotype frequency } & \multirow[b]{2}{*}{$P$ value } & \multirow[b]{2}{*}{ FDR } \\
\hline & Genotype & RSTR (case) & LTBI (control) & $\mathrm{OR}^{\mathrm{A}}(95 \% \mathrm{Cl})$ & & \\
\hline \multirow{3}{*}{ rs10480299 } & $\mathrm{T} / \mathrm{T}$ & 0.311 & 0.556 & $2.78(1.77-4.38)$ & $1.00 \times 10^{-5}$ & $2.6 \times 10^{-3}$ \\
\hline & $T / C$ & 0.500 & 0.407 & & & \\
\hline & $c / C$ & 0.189 & 0.037 & & & \\
\hline \multirow[t]{3}{*}{ rs10480300 } & $C / C$ & 0.500 & 0.693 & $2.48(1.51-4.05)$ & $3.16 \times 10^{-4}$ & $2.7 \times 10^{-2}$ \\
\hline & $\mathrm{C} / \mathrm{T}$ & 0.378 & 0.291 & & & \\
\hline & $T / T$ & 0.122 & 0.016 & & & \\
\hline \multirow[t]{3}{*}{ rs114166988 } & $T / T$ & 0.554 & 0.751 & $2.64(1.56-4.49)$ & $3.18 \times 10^{-4}$ & $2.7 \times 10^{-2}$ \\
\hline & $T / C$ & 0.351 & 0.243 & & & \\
\hline & $C / C$ & 0.095 & 0.005 & & & \\
\hline \multirow[t]{3}{*}{ rs10224002 } & $A / A$ & 0.274 & 0.439 & $1.94(1.29-2.91)$ & $1.40 \times 10^{-3}$ & $9.0 \times 10^{-2}$ \\
\hline & $A / G$ & 0.425 & 0.433 & & & \\
\hline & $\mathrm{G} / \mathrm{G}$ & 0.301 & 0.128 & & & \\
\hline \multirow[t]{3}{*}{ rs56145758 } & $T / T$ & 0.757 & 0.905 & $3.22(1.51-6.88)$ & $2.56 \times 10^{-3}$ & 0.12 \\
\hline & $\mathrm{T} / \mathrm{A}$ & 0.216 & 0.095 & & & \\
\hline & $A / A$ & 0.027 & 0.000 & & & \\
\hline \multirow[t]{3}{*}{ rs115310513 } & $A / A$ & 0.757 & 0.894 & $2.98(1.46-6.08)$ & $2.69 \times 10^{-3}$ & 0.12 \\
\hline & $A / G$ & 0.203 & 0.106 & & & \\
\hline & G/G & 0.041 & 0.000 & & & \\
\hline \multirow[t]{3}{*}{ rs1860746 } & $C / C$ & 0.541 & 0.693 & $2.27(1.31-3.93)$ & $3.51 \times 10^{-3}$ & 0.12 \\
\hline & $C / A$ & 0.392 & 0.291 & & & \\
\hline & $A / A$ & 0.068 & 0.016 & & & \\
\hline
\end{tabular}

${ }^{A} \mathrm{OR}$ indicates additive odds for being a case (RSTR) compared with control (LTBI) for each copy of the minor allele inherited (additive model).

may relate to the association of diabetes mellitus with Mtb disease susceptibility (65). While our transcriptional data identify expression patterns in metabolic pathways and not metabolite levels, serum profiling of OA and PA levels in RSTR versus LTBI subjects remains of future interest.

The specific mechanism by which PA enhances intracellular Mtb growth remains to be identified. PA is known to inhibit autophagy by limiting AMPK-mediated activation of ULK1 as measured by autophagosome formation, microtubule-associated protein 1 light chain 3 (LC3) lipidation and LC3 puncta formation (37). Although the enhanced intracellular Mtb growth in PA-treated cells was maintained in ULK1-deficient THP-1 cells (Figure 6B), a role for PA in modulating autophagy has not been excluded considering AMPK activation restored growth restriction (Figure 6A) and is known to activate ULK1 and ULK2, which have the redundant function in autophagosome initiation (66). Alternatively, LC3-associated phagocytosis (LAP) is independent of ULK1 and could be modulated by PA (67). Our data also remain consistent with a host-protective role for AMPK activation in reversing the deleterious effects that PA has on separate

elevated among Mtb household contacts in the Gambia who converted TST/IGRA testing as compared with nonconverters, both initially after exposure and after 3 months of follow-up (11). Host metabolomic signatures that distinguish subjects with Mtb disease versus healthy contacts (58) and that predict progression from LTBI to active Mtb disease (59) also have included medium- and longchain FFAs. Lipid metabolism lies at an interface between host and pathogen responses within macrophages (60). Mtb acquires host lipids and cholesterol as carbon sources (61-63) and access to these nutrients may be restricted as part of the IFN- $\gamma$-primed host response (64). Furthermore, virulent Mtb shifts mitochondrial dependency from glucose to exogenous fatty acids in primary human macrophages whereas avirulent or dead Mtb promotes glycolytic flux (20), suggesting these macrophage metabolic switches may be linked to virulence of the invading pathogen. We identified multiple independent macrophage gene programs downstream of OA (and linoleic/lauric acid) stimulation that strongly enriched among RSTR monocyte transcriptomes, whereas a single gene set (Set 7) that enriched among LTBI subjects correlates with the macrophage transcriptional response to PA (33). This dichotomy of gene programs between RSTR and LTBI monocytes parallels the opposing biologic effects of OA and PA, which are among the most abundant circulating FFAs. Since PA treatment of healthy donor macrophages enhanced intracellular Mtb replication, our combined results suggest that host-protective pathways are negatively influenced by PA. Interestingly, insulin sensitivity is also negatively impacted by an elevated plasma PA/OA ratio (34), an effect that pathways that may impact Mtb control, such as increased ceramide synthesis/lipotoxicity $(68,69)$ and resultant ER stress $(70,71)$; mitochondrial dysfunction, including the generation of reactive oxygen species (ROS) $(72,73)$ and the disruption of FFA beta-oxidation (74); or effects on macrophage polarization and cytokine responses $(75,76)$. Many of these PA effects are antagonized by monounsaturated fatty acids including OA (34, 68, 76-78), which correlates with our RSTR versus LTBI transcriptional dichotomy. While prior studies using differentiated THP-1 cells found that preinfection OA treatment restricted intracellular Mtb (79), we found no OA effect on MDMs treated immediately after infection. Parihar et al. (80) also reported that pretreatment of murine bone marrow-derived macrophages with OA restricted intracellular Mtb growth, whereas PA had minimal effects at high doses $(500 \mu \mathrm{M})$, suggesting our findings may be specific to human primary macrophages and highlighting the potential importance of the timing of FFA treatments.

We found that both PA and OA modulated host cytokine responses following Mtb whole cell lysate stimulation whereas only PA impacted these responses within Mtb-infected cells (Figure 5). Strikingly, PA had opposing effects that were dependent on Mtb viability where proinflammatory cytokine secretion increased after Mtb whole cell lysate stimulation but decreased in Mtb-infected cells. Under cellular conditions that promote glycolysis in macrophages (e.g., after TLR stimulation), IL1B transcription increases resulting in host-protective effects like CFU reduction (21). Recent work demonstrates that live Mtb attenuates $I L 1 B$ transcription 
(relative to heat-killed Mtb) by exploiting a host pathway that negatively regulates glycolysis (81). Our results are consistent with a mechanism whereby PA and viable Mtb interact to restrict the IL-1 $\beta$ response at the transcriptional level, even as PA enhances TLR-induced IL-1 $\beta$ via posttranslational NLRP3 activation (Figure $5 \mathrm{~A}$ and ref. 37). Further studies are required to demonstrate whether this PA-Mtb interaction results in modulation of glycolysis or other metabolic pathways to restrict proinflammatory cytokine secretion.

Our study has several limitations, including those related to LTBI diagnostics that define the RSTR phenotype. Mechanisms that underly resistance to TST/IGRA conversion could reflect (a) inadequate exposure, (b) early clearance of the pathogen via innate immune cell functions that precede $\mathrm{T}$ cell priming (10, $82)$, (c) clearance of Mtb through antigen-specific $\mathrm{CD} 4^{+} \mathrm{T}$ cell or other adaptive responses that are independent of IFN- $\gamma(4)$, or (d) persistent paucibacillary infection that fails to prime IFN- $\gamma$-producing, antigen-specific $\mathrm{T}$ cell responses but otherwise may be indistinguishable from LTBI. Emerging evidence from the same Ugandan RSTR cohort studied here indicates these subjects are indeed Mtb-exposed as determined by both epidemiologic parameters $(7,8)$ and alternative (IFN- $\gamma$-independent) immune sensitization (4). In addition, our analyses identified transcriptional differences in unstimulated RSTR and LTBI monocytes. Whether this extends to differences in metabolite levels in vivo or changes in metabolic flux after ex vivo Mtb infection is an important focus of future studies. Transcriptional differences in unstimulated monocytes may suggest distinct epigenetic programing that can be specifically evaluated using existing platforms (e.g., methylation profiling, ATACseq). The transcriptional differences identified here in peripheral blood also incentivize the collection of alveolar macrophages; these cells are the earliest targets of Mtb in vivo, with distinct metabolic activities and the potential to dramatically influence innate and cellular responses that distinguish RSTR and LTBI outcomes. Similarly, it will be important to confirm whether the PA effect on Mtb replication within MDMs is also seen in other primary myeloid cell types, including alveolar macrophages and dendritic cells that have distinct ontogeny or differentiation pathways that may involve metabolic programming (22).

In summary, we report that transcriptional programs are distinct in monocytes from RSTR and LTBI donors. These differences, especially those conserved across 2 distinct RSTR populations, give insight into the early Mtb response in subjects who resist TST and IGRA conversion and implicate distinct metabolic programs. Furthermore, we identify polymorphisms in the central metabolic regulator AMPK that are associated with RSTR individuals. Additional work is required to define the protective mechanisms of these metabolic pathways in hopes they may be amplified by host-directed therapies that could augment current antimicrobial therapy.

\section{Methods}

Subject recruitment. Our study involved ex vivo characterization of whole blood and monocytes isolated from highly Mtb-exposed, HIV-negative donors in Uganda and South Africa. We hypothesized that donors who resisted TST/IGRA conversion would have distinct transcriptional signatures as compared with control donors with LTBI and that these differences may inform mechanistic hypotheses. Ugandan household contacts of index cases with culture-confirmed pulmonary TB were recruited between 2002 to 2012 as part of the Kawempe Community Health Study (Kampala, Uganda) for serial TST testing (8), and a subset of these household contacts were successfully recontacted after 8 to 10 years for PBMC collection and to complete serial IGRA and repeat TST testing (7).

The Highly Exposed TB Uninfected (HETU) study was a prospective, longitudinal study of HIV-negative gold miners who were screened between August 2015 and December 2016 in North West Province, South Africa (our unpublished observations). Subjects who had worked in the mining industry for 15 years or more, were aged 33 to 60 years, had no history, symptoms, nor prior treatment of TB, and no silicosis were eligible. Subjects underwent Quantiferon-TB Gold In-Tube (QFT-GIT, Qiagen) and TST (Matoux method, 5 tuberculin units of PPD, Statens Serum Institute) evaluations at baseline. RSTR gold miners had concordant negative TST $(<5 \mathrm{~mm}$ in duration) and negative QFT-GIT (as defined by manufacturer) testing at enrollment. At enrollment, PBMCs were collected and isolated by Ficoll gradient, cryopreserved, and shipped to Seattle, Washington, USA, along with whole blood frozen in PAXgene RNA tubes (Qiagen).

Details, including RSTR case definitions, are published for the Ugandan cohort $(7,8)$ and are available in Supplementary Materials.

Cell culture, reagents, and mycobacterial strains. Roswell Park Memorial Institute 1640 medium (RPMI 1640, Gibco) was supplemented with FBS (Atlas Biologicals) to a final concentration of $5 \%$ (RPMI-5) or 10\% (RPMI-10). Macrophage colony-stimulating factor (M-CSF) was purchased from Peprotech. PA (catalog P5585), OA (catalog O1383), and fatty-acid free BSA (catalog A7030) were purchased from MilliporeSigma. A 5\% (wt/vol) BSA solution in 1× PBS (Gibco) was filter-sterilized and stored up to 3 months at $4^{\circ} \mathrm{C}$ and used to solubilize FFAs. The endotoxin content of this solution was negligible $(<0.2 \mathrm{pg}$ endotoxin per $1 \mathrm{mg} \mathrm{BSA})$ as confirmed by limulus assay (Lonza). The AMPK activator “A 769669" was purchased (Tocris Bioscience), reconstituted in DMSO $(50 \mathrm{mM})$, and aliquots were stored $\left(-80^{\circ} \mathrm{C}\right)$ until final dilutions were prepared in RPMI $/ 5$.

U937 cells (ATCC CLR1593) with disruptions in NLRP3 and NLRC4 were generated using CRISPR/Cas9 by lentivirus delivery of guide RNAs (Supplementary Materials), selected in puromycin (83) as described for ULK1 (84), and screened phenotypically and by sequencing. Prior to each experiment, these U937 cells were differentiated for 72 hours in phorbol 12-myristate 13-acetate (PMA, Invitrogen), washed, and rested overnight in RPMI/10 without PMA before infection or stimulation.

The Mycobacterium tuberculosis Erdman reporter strain expressing the luxCDABE operon from Vibrio harveyi (Mtb-lux) was a gift of Jeff Cox (University of California at Berkeley) and was grown in Middlebrook 7H9 media supplemented with glycerol (Fisher; $4 \mathrm{~mL} / \mathrm{L}$ ), albumin-dextrose-catalase (ADC) supplement (Middlebrook, $100 \mathrm{~mL} / \mathrm{L}$ ) and Tween 80 (Fisher; 0.05\% final). We previously demonstrated a linear correlation between relative light units (RLUs) and colony forming units (CFUs) using this strain (85).

Monocyte isolation, RNA isolation. Cryopreserved PBMCs were thawed, washed, and resuspended at 2 million cells per milliliter in RPMI-10 supplemented with M-CSF (50 ng/mL), then rested overnight. $\mathrm{CD} 14^{+}$monocytes were isolated by magnetic bead column purification using negative selection (Monocyte Isolation Kit II, Miltenyi Biotec) and then plated at 1 million cells per milliliter RPMI-10 supplemented with M-CSF in duplicate wells. After 24 hours ( 48 hours total $\mathrm{M}-\mathrm{CSF}$ exposure), media were removed and cells were lysed in Trizol 
(Invitrogen) and frozen. The aqueous phase of thawed lysates were then collected after chloroform extraction to which $100 \%$ ethanol was added before applying to miRNeasy micro columns for RNA isolation according to the manufacturer's instructions (Qiagen). PAXgene tubes were thawed at room temperature and RNA was isolated using PAXgene miRNA spin columns (Qiagen). Eluates were globin-depleted by oligonucleotide hybridization according to the manufacturer's instructions (Ambion). RNA from both PAXgene and monocyte samples was quantitated by Nanodrop (Thermo Fisher Scientific) and quality was assessed ( $\mathrm{RIN} \geq 8.0$ ) by Agilent TapeStation.

RNA sequencing and data processing. cDNA libraries were prepared using random hexamer priming and rRNA depletion according to the manufacturer's specifications (Takara, SMARTer RNAseq Kit-Pico Input Mammalian). RNA sequencing was performed on the Illumina HiSeq 2500 in high output mode, which achieved a read depth of 30 million paired-end, 50 base pair reads. Adapters were removed with Cutadapt (86) prior to sequence alignment using STAR 2.6.0a (87) with the GRCh38 reference genome. Counts for each gene were assigned using RSEM 1.3.0 (88). Data were transformed with respect to library size using voom (89). Using multidimensional scaling (MDS), we found no evidence of batch effects nor any global influence of age or the other available metadata from each cohort when plotting the first 2 dimensions. In Uganda, these included family group, BMI, epidemiologic risk score, and BCG history (Supplemental Figure 4). In South Africa, these included country of origin, occupation (skilled versus unskilled labor), living arrangement (mine hostel or not), active or accumulative years worked underground, and ethnicity (Supplemental Figure 5). However, ethnicity was associated with RSTR versus LTBI status (Supplemental Tables 2 and 6; $P=0.003$ ). Similarly, occupation level was also associated with ethnicity where skilled laborers were significantly more likely to be white $\left(P<4.8 \times 10^{-5}\right)$. Since the association between white ethnicity and RSTR status most likely reflects lower exposure among skilled as compared with unskilled laborers, all analyses were performed with and without inclusion of $(n=6)$ White subjects (Figure 1). Although our main findings were unchanged, greater overlap existed across Ugandan and South African signatures using this stratified analysis so these analyses are presented.

Gene set enrichment analysis. To identify enrichment of biologic pathways or macrophage activation states with the RSTR or LTBI clinical phenotypes, we used the Correlation Adjusted Mean Rank gene set test (CAMERA) implemented in limma R package version 3.42.0 (30), which is a competitive gene set test that is similar to GSEA (31), but less susceptible to inter-gene correlations. We performed separate analyses for each of 3 lists of gene sets: MSigDB (32) C2 and C7 (9547 gene sets), MSigDB C5 gene sets $(n=5914), 49$ transcriptional modules identified by Xue et al. (modules 1-49; ref. 33). Nominal $P$ values were calculated for the degree of enrichment for individual gene sets using CAMERA, which were then adjusted (FDR) to account for simultaneous comparisons. Details are available in Supplementary Materials.

Preparation of free fatty acids. PA and OA were solubilized by complexation with 5\% BSA (MilliporeSigma) prepared in $1 \times$ PBS (Gibco) in a previously described method (90). These resulting 4.6 mM FFA/BSA solutions were diluted further to desired final concentrations in warm RPMI-5 and filter sterilized through a $0.2 \mu \mathrm{m}$ PES membrane (Millipore). Molar ratios of the $4.6 \mathrm{mM}$ FFA/BSA complexes were experimentally confirmed to be 3:1 using a nonesterified fatty acid-HR quantitation kit (FUJIFILM Wako Diagnos- tics) and BCA assay (Thermo Fisher Scientific). After dilution of FFA/BSA complexes in RMPI-5, the ratio for each $200 \mu \mathrm{M}$ solution was measured to be 1.2:1.

Intracellular monocyte-derived macrophage replication. Human MDMs were prepared from LRS TRIMA chambers harvested from healthy donors (Bloodworks Northwest). CD14+ monocytes were isolated and differentiated in RPMI-10 plus $50 \mathrm{ng} / \mathrm{mL} \mathrm{M}$-CSF for a total of 6 to 7 days prior to infection. Log-phase (OD $<0.6)$ Mtb-lux cultures were applied to a $5-\mu \mathrm{m}$ syringe filter (Millipore) to achieve a single-cell suspension, diluted in RPMI, and added to MDM cultures (MOI = 1-2 CFU per cell). Following a 4-hour infection, monolayers were washed twice with warm RPMI, and then returned to fresh RPMI-5 media containing M-CSF and the indicated doses of FFA or controls matched for BSA content. Luminescence was recorded using a Synergy H4 multimode microplate reader (Biotek Instruments). PMA-differentiated U937 cells were similarly infected, then treated with $200 \mu \mathrm{M}$ PA.

Cytokine secretion. Human MDMs were prepared from LRS TRIMA chambers as above, treated overnight with FFAs, and then stimulated with Mtb (H37Rv) whole cell lysate $(25 \mu \mathrm{g} / \mathrm{mL}$; BEI Resources), live Mtb-lux filtered as above (MOI = $5 \mathrm{CFU}$ per cell), or media control in the presence of FFA. Supernatants were harvested at 24 hours after stimulation and those containing infectious Mtb were filtered to remove them from BSL-3, after which cytokine concentrations were assayed by DuoSet ELISA (R\&D Systems) using TMB peroxidase substrate (SeraCare) and read on a SpectraMax-Plus plate reader (Molecular Devices).

Macrophage viability assay. Human MDM viability after PA, OA, and BSA vehicle control was quantitated using the IncuCyte live cell analysis system (Sartorius). Healthy donor MDMs were treated with the indicated final concentrations of each fatty acid and Sytox Green nucleic acid stain diluted according to the manufacturer's specifications (Thermo Fisher Scientific) and immediately imaged over 72 hours. Cell death was quantitated as the proportion of cells with nuclear green fluorescence divided by the average Syto Green-positive (Thermo Fisher Scientific) cells stained in control wells. Fluorescence signal decayed after approximately 48 hours, so the peak signal was used to estimate cell death (\%).

AMPK isoform candidate gene association study. Using the Illumina $\mathrm{MEGA}^{\mathrm{EX}}$ array consisting of 2 million single nucleotide polymorphisms (SNPs), we genotyped HIV-negative Ugandan household contacts defined as RSTR $(n=74)$ or LTBI $(n=189)$ as previously described (7). This array selected variants optimized for samples of African ancestry. Gene regions of the 7 isoforms encoding each subunit of AMPK (PRKAA1, PRKAA2, PRKAB1, PRKAB2, PRKAG1, PRKAG2, PRKAG3) were examined along with $5 \mathrm{~kb}$ flanking sequences defined using Ensembl (build GRCh37). Among these regions, there were a total of 257 SNPs included in the Illumina MEGA ${ }^{\mathrm{EX}}$ array (12 in PRKAA1, 20 in PRKAA2, 2 in PRKAB1, 13 in PRKAB2, 2 in PRKAG1, 201 in PRKAG2, 7 in PRKAG3). We compared the genotype frequency in cases (RSTR) and controls (LTBI) with an additive model, accounting for family relationships via a penalized quasi-likelihood approximation to the generalized linear mixed model (GENESIS), and adjusted for age and sex $(52,53,91,92)$. To adjust for multiple comparisons, $P$ values were adjusted by FDR. For SNPs of interest $(P<0.01)$, we also examined dominant and recessive models.

Flow cytometry. Peripheral blood mononuclear cells from Ugandan donors (23 RSTR and 23 LTBI) were stained for viability with LIVE/ 
DEAD Fixable Dead Cell Stain (Thermo Fisher Scientific), permeabilized and fixed in FACS Permeabilizing Solution 2 (BD Biosciences), before staining for CD56, washed, then stained with the remaining antibody cocktail (Supplemental Table 15). Cells were washed, treated with $2 \mathrm{mM}$ EDTA $/ 1 \%$ PFA at $4^{\circ} \mathrm{C}$, washed twice, then resuspended in $2 \mathrm{mM}$ EDTA/1 $\times$ PBS at $4^{\circ} \mathrm{C}$ overnight. Cell counts were acquired on a LSRFortessa (BD Biosciences) and cell population frequencies were calculated using the indicated ratios. Differences in mean frequencies were compared (Mann-Whitney $U$ test).

Statistics. For differential gene expression between RSTR and LTBI phenotypes, nominal $P$ values were calculated and corrected for FDR by the Benjamini-Hochberg procedure, as implemented by the $\mathrm{R}$ package limma. FFA dose-dependent effects on Mtb growth were compared by fitting RLU data to a simple linear model using Prism 8.3.1 (GraphPad Software, LLC). Data were log-transformed where indicated to achieve the best regression fit. Ordinary 1-way ANOVA was used to confirm a significant dose-dependent A 769662 inhibitory effect (Figure 6A) where $P$ values were calculated for each pairwise comparison and adjusted using Tukey's multiple comparison test. For single-dose pairwise comparisons (Figure 6B), $P$ values were instead calculated by Student's 2-tailed $t$ test. Study subject epidemiologic and demographic data were compared using Stata/IC 15.1 (StataCorp LLC). $P$ values were calculated using the $\chi^{2}$ or Fisher's exact tests for categorical variables and the Mann-Whitney $U$ test for continuous variables. A $P$ value less than 0.05 was considered significant.

Study approval. For both the Ugandan and South African cohorts, written informed consent was provided by all subjects, or for illiterate participants, witnessed verbal consent was obtained. Study protocols were approved by respective institutional review boards for the Ugandan (the National AIDS Research Committee, The Uganda National Council on Science and Technology, and the IRBs at the University Hospitals Cleveland Medical Center and the University of Washington) and for South African cohorts (the ethics committees of the University of Witwatersrand, London School of Hygiene \& Tropical Medicine and the University of Washington).

Data and materials transfer agreements. Raw transcriptomic data are available upon request, but require authorization by data access committees (DACs). To request access, please contact the following representatives for each DAC: Sudha Iyenhgar (ski@case.edu) for Ugandan transcriptomic data access and Salome Charalambous (scharalambous@auruminstitute.org) for South African transcriptomic data access. Once access is approved, the files will be available on the NCBI database of Genotypes and Phenotypes (dbGaP) Data Browser under accession 002445.v1.p1 (https://www.ncbi.nlm.nih.gov/gap/).

\section{Author contributions}

All experiments were performed by JDS, GJP, FKN, AR, and AD Graustein, with input on design from CS and TRH. Computational analyses were performed by PTV and RG. Statistical genetic analysis in the Ugandan cohort was conducted by PB and
CMS. The manuscript was written by JDS and TRH. All authors reviewed the manuscript. Clinical cohorts were established in Uganda with initial epidemiologic characterization by CMS, $\mathrm{HMK}$, and WHB. Study coordination and epidemiologic characterization in South Africa were accomplished by VC, KV, KLF, AD Grant, TN, PM, RSW, and GC.

\section{Acknowledgments}

We would like to acknowledge LaShaunda Malone, Keith Chervenak, and Marla Manning for their efforts coordinating the Uganda studies, including database and program management. Furthermore, we acknowledge all clinical and research staff integral to the Ugandan household contact study-Mary Nsereko, Alphonse Okwera, Moses Joloba, Hussein Kisingo, Sophie Nalukwago, Dorcas Lamunu, Deborah Nsamba, Annet Kawuma, Saidah Menya, Joan Nassuna, Joy Beseke, Michael Odie, Henry Kawoya, Shannon Pavsek, E. Chandler Church, Anna Duewiger, and Bonnie Thiel-as well as the participation of the individual study subjects and their families.

We also would like to acknowledge the North West Department of Health and gold mining companies, the participants of the HETU study, and the following lab and field researchers: Theunius Cloete, Ithabeleng Morojele, Keolebogile Ntshamane, Tebogo Rampai, Ndumiso Sithole, Zamakhabako Mhlanga, Ntombomzi Motsoeneng, James Seseng, Samuel Tlhabakwane, Abel Qas, Kabelo Leshoro, Samantha Naicker, Lindiwe Nhlangulela, Mandla Mlotshwa, Robyn Beater, Martha Albani, Ken Clarke, Palesa Mosweu, Letlhogonolo Seabela, and Nondumiso Langa.

Library construction, RNA sequencing, and sequence alignments were performed at the Fred Hutchinson Cancer Research Center by Matthew Fitzgibbon and Jeff Delrow. We thank Kim Dill-McFarland for advice on bioinformatic analyses and Alan Chait and Chang Han for their advice on fatty acid complex preparations. Cytotoxicity data using the IncuCyte instrument were accessed with the help of Andrew Oberst and Cassidy Hagan.

This work was funded by grants from the US National Institutes of Health grants K08AI143926 and T32AI007044 (to JDS), R01AI124348 (to WHB, TRH, CMS, HMK, GC, RSW, VC, RG, and CS), U01AI115642 (to WHB, TRH, CMS, and HMK), K24AI137310 (to TRH), and contract number NO1AI70022 (WHB, TRH, CMS, and HMK); the Bill and Melinda Gates Foundation grant OPP1151836 (to TRH, WHB, CMS, HMK, CS, GC, and RSW); and the South African Medical Research Council grant ACT4TB/HIV (to GC and RSW).

Address correspondence to: Jason D. Simmons, University of Washington, 750 Republican St., Seattle, Washington 98109, USA. Phone: 206.685.6383; Email: jasonds@uw.edu.
1. World Health Organization. Global Tuberculosis Report 2019. https://apps.who.int/iris/handle/10665/329368. Updated October 15, 2019. Accessed June 9, 2021.

2. Houben RM, Dodd PJ. The global burden of latent tuberculosis infection: a re-estimation using mathematical modelling. PLoS Med. 2016;13(10):e1002152.

3. Simmons JD, et al. Immunological mechanisms of human resistance to persistent Mycobacterium tuberculosis infection. Nat Rev Immunol. 2018;18(9):575-589.
4. Lu LL, et al. IFN-gamma-independent immune markers of Mycobacterium tuberculosis exposure. Nat Med. 2019;25(6):977-987.

5. Mave V, et al. Infection free "resisters" among household contacts of adult pulmonary tuberculosis. PLoS One. 2019;14(7):e0218034 
6. Medawar L, et al. Analysis of cellular and soluble profiles in QuantiFERON nonconverters, converters, and reverters in the Gambia. Immun Inflamm Dis. 2019;7(4):260-270.

7. Stein CM, et al. Long-term stability of resistance to latent mycobacterium tuberculosis infection in highly exposed tuberculosis household contacts in Kampala, Uganda. Clin Infect Dis. 2019;68(10):1705-1712.

8. Stein CM, et al. Resistance and susceptibility to mycobacterium tuberculosis infection and disease in tuberculosis households in Kampala, Uganda. Am J Epidemiol. 2018;187(7):1477-1489.

9. Verrall AJ, et al. Early clearance of Mycobacterium tuberculosis: The INFECT case contact cohort study in Indonesia. J Infect Dis. 2020;221(8):1351-1360.

10 . Verrall AJ, et al. Early clearance of Mycobacterium tuberculosis is associated with increased innate immune responses. JInfect Dis. 2020;221(8):1342-1350.

11. Weiner J, et al. Changes in transcript, metabolite, and antibody reactivity during the early protective immune response in humans to mycobacterium tuberculosis infection. Clin Infect Dis. 2020;71(1):30-40.

12. Vorkas CK, et al. Mucosal-associated invariant and $\gamma \delta$ T cell subsets respond to initial Mycobacterium tuberculosis infection. JCI Insight. 2018;3(19):e121899.

13. Cohen SB, et al. Alveolar macrophages provide an early Mycobacterium tuberculosis niche and initiate dissemination. Cell Host Microbe. 2018;24(3):439-446.

14. Delahaye JL, et al. Cutting edge: Bacillus Calmette-Guérin-induced T cells shape Mycobacterium tuberculosis infection before reducing the bacterial burden. J Immunol. 2019;203(4):807-812.

15. Wolf AJ, et al. Mycobacterium tuberculosis infects dendritic cells with high frequency and impairs their function in vivo. J Immunol. 2007;179(4):2509-2519.

16. Churchyard GJ, et al. A trial of mass isoniazid preventive therapy for tuberculosis control. $\mathrm{N} \mathrm{EnglJ}$ Med. 2014;370(4):301-310.

17. Hanifa Y, et al. Prevalence of latent tuberculosis infection among gold miners in South Africa. Int J Tuberc Lung Dis. 2009;13(1):39-46.

18. Vynnycky E, et al. Tuberculosis control in South African gold mines: mathematical modeling of a trial of community-wide isoniazid preventive therapy. Am JEpidemiol. 2015;181(8):619-632.

19. Cheng CY, et al. Metabolic energy sensors as targets for designing host-directed therapies for tuberculosis. JLeukoc Biol. 2018;103(2):215-223.

20. Cumming BM, et al. Mycobacterium tuberculosis induces decelerated bioenergetic metabolism in human macrophages. Elife. 2018;7:e39169.

21. Gleeson LE, et al. Cutting edge: Mycobacterium tuberculosis induces aerobic glycolysis in human alveolar macrophages that is required for control of intracellular bacillary replication. J Immunol. 2016;196(6):2444-2449.

22. Huang L, et al. Growth of Mycobacterium tuberculosis in vivo segregates with host macrophage metabolism and ontogeny. J Exp Med. 2018;215(4):1135-1152.
23. Ouimet M, et al. Mycobacterium tuberculosis induces the miR-33 locus to reprogram autophagy and host lipid metabolism. Nat Immunol. 2016;17(6):677-686.

24. Singh V, et al. Mycobacterium tuberculosis-driven targeted recalibration of macrophage lipid homeostasis promotes the foamy phenotype. Cell Host Microbe. 2012;12(5):669-681.

25. Berry MP, et al. An interferon-inducible neutrophil-driven blood transcriptional signature in human tuberculosis. Nature. 2010;466(7309):973-977.

26. Singhania A, et al. A modular transcriptional signature identifies phenotypic heterogeneity of human tuberculosis infection. Nat Commun. 2018;9(1):2308.

27. Sweeney TE, et al. Genome-wide expression for diagnosis of pulmonary tuberculosis: a multicohort analysis. Lancet Respir Med. 2016;4(3):213-224.

28. Zak DE, et al. A blood RNA signature for tuberculosis disease risk: a prospective cohort study. Lancet. 2016;387(10035):2312-2322.

29. Vallania F, et al. Leveraging heterogeneity across multiple datasets increases cell-mixture deconvolution accuracy and reduces biological and technical biases. Nat Commun. 2018;9(1):4735.

30. Wu D, Smyth GK. Camera: a competitive gene set test accounting for inter-gene correlation. Nucleic Acids Res. 2012;40(17):e133.

31. Subramanian A, et al. Gene set enrichment analysis: a knowledge-based approach for interpreting genome-wide expression profiles. Proc Natl Acad Sci U S A. 2005;102(43):15545-15550.

32. Liberzon A, et al. The Molecular Signatures Database (MSigDB) hallmark gene set collection. Cell Syst. 2015;1(6):417-425.

33. Xue J, et al. Transcriptome-based network analysis reveals a spectrum model of human macrophage activation. Immunity. 2014;40(2):274-288.

34. Palomer X, et al. Palmitic and oleic acid: the yin and yang of fatty acids in type 2 diabetes mellitus. Trends Endocrinol Metab. 2018;29(3):178-190.

35. Kanetsuna F. Bactericidal effect of fatty acids on mycobacteria, with particular reference to the suggested mechanism of intracellular killing. Microbiol Immunol. 1985;29(2):127-141.

36. Meikle V, et al. A protein complex from human milk enhances the activity of antibiotics and drugs against Mycobacterium tuberculosis. Antimicrob Agents Chemother. 2019;63(2):e01846-18.

37. Wen $\mathrm{H}$, et al. Fatty acid-induced NLRP3-ASC inflammasome activation interferes with insulin signaling. Nat Immunol. 2011;12(5):408-415.

38. Steinberg GR, Carling D. AMP-activated protein kinase: the current landscape for drug development. Nat Rev Drug Discov. 2019;18(7):527-551.

39. Kumar R, et al. Immunometabolism of phagocytes during Mycobacterium tuberculosis infection. Front Mol Biosci. 2019;6:105.

40. Pajuelo D, et al. NAD hydrolysis by the tuberculosis necrotizing toxin induces lethal oxidative stress in macrophages. Cell Microbiol. 2020;22(1):e13115.

41. Russell DG, et al. Foamy macrophages and the progression of the human tuberculosis granuloma. Nat Immunol. 2009;10(9):943-948.

42. Shi L, et al. Infection with Mycobacterium tuberculosis induces the Warburg effect in mouse lungs. Sci Rep. 2015;5:18176.

43. Singhal A, et al. Metformin as adjunct antituberculosis therapy. Sci Transl Med. 2014;6(263):263ra159.

44. Mehrotra P, et al. Pathogenicity of Mycobacterium tuberculosis is expressed by regulating metabolic thresholds of the host macrophage. PLOS Pathog. 2014;10(7):e1004265.

45. Rodriguez-Prados JC, et al. Substrate fate in activated macrophages: a comparison between innate, classic, and alternative activation. JImmunol. 2010;185(1):605-614.

46. Tannahill GM, et al. Succinate is an inflammatory signal that induces IL-1 $\beta$ through HIF-1 $\alpha$. Nature. 2013;496(7444):238-242.

47. Braverman J, et al. HIF-1 $\alpha$ is an essential mediator of IFN- $\gamma$-dependent immunity to Mycobacterium tuberculosis. J Immunol. 2016;197(4):1287-1297.

48. Thompson EG, et al. Host blood RNA signatures predict the outcome of tuberculosis treatment. Tuberculosis (Edinb). 2017;107:48-58.

49. Stein CM, et al. Genome scan of M. tuberculosis infection and disease in Ugandans. PLoS One. 2008;3(12):e4094.

50. Cobat A, et al. Two loci control tuberculin skin test reactivity in an area hyperendemic for tuberculosis. JExp Med. 2009;206(12):2583-2591.

51. Cobat A, et al. Tuberculin skin test negativity is under tight genetic control of chromosomal region 11p14-15 in settings with different tuberculosis endemicities. JInfect Dis. 2015;211(2):317-321.

52. Igo RP Jr, et al. Fine-mapping analysis of a chromosome 2 region linked to resistance to Mycobacterium tuberculosis infection in Uganda reveals potential regulatory variants. Genes Immun. 2019;20(6):473-483.

53. Hall NB, et al. Polymorphisms in TICAM2 and IL1B are associated with TB. Genes Immun. 2015;16(2):127-133.

54. Cheng CY, et al. Host sirtuin 1 regulates mycobacterial immunopathogenesis and represents a therapeutic target against tuberculosis. Sci Immunol. 2017;2(9):eaaj1789.

55. Yang CS, et al. The AMPK-PPARGC1A pathway is required for antimicrobial host defense through activation of autophagy. Autophagy. 2014;10(5):785-802.

56. Leow MK, et al. Latent tuberculosis in patients with diabetes mellitus: prevalence, progression and public health implications. Exp Clin Endocrinol Diabetes. 2014;122(9):528-532.

57. Magee MJ, et al. Reduced prevalence of latent tuberculosis infection in diabetes patients using metformin and statins. Eur Respir J. 2019;53(3):1801695.

58. Weiner J, et al. Biomarkers of inflammation, immunosuppression and stress with active disease are revealed by metabolomic profiling of tuberculosis patients. PLoS One. 2012;7(7):e40221.

59. Weiner J, et al. Metabolite changes in blood predict the onset of tuberculosis. Nat Commun. 2018;9(1):5208.

60. Chandra P, et al. Inhibition of fatty acid oxidation promotes macrophage control of Mycobacterium tuberculosis. mBio. 2020;11(4):e01139-20.

61. Daniel J, et al. Mycobacterium tuberculosis uses host triacylglycerol to accumulate lipid droplets and acquires a dormancy-like pheno- 
type in lipid-loaded macrophages. PLoS Pathog. 2011;7(6):e1002093.

62. Lee W, et al. Intracellular Mycobacterium tuberculosis exploits host-derived fatty acids to limit metabolic stress. J Biol Chem. 2013;288(10):6788-6800.

63. Pandey AK, Sassetti CM. Mycobacterial persistence requires the utilization of host cholesterol. Proc Natl Acad Sci U S A. 2008;105(11):4376-4380.

64. Knight M, et al. Lipid droplet formation in Mycobacterium tuberculosis infected macrophages requires IFN- $\gamma /$ HIF- $1 \alpha$ signaling and supports host defense. PLoS Pathog. 2018;14(1):e1006874.

65. Al-Rifai RH, et al. Association between diabetes mellitus and active tuberculosis: A systematic review and meta-analysis. PLoS One. 2017;12(11):e0187967.

66. McAlpine F, et al. Regulation of nutrient-sensitive autophagy by uncoordinated 51-like kinases 1 and 2. Autophagy. 2013;9(3):361-373.

67. Koster S, et al. Mycobacterium tuberculosis is protected from NADPH oxidase and LC3-associated phagocytosis by the LCP protein CpsA. Proc Natl Acad Sci U S A. 2017;114(41):E8711-E8720.

68. Hu W, et al. Differential regulation of dihydroceramide desaturase by palmitate versus monounsaturated fatty acids: implications for insulin resistance. JBiol Chem. 2011;286(19):16596-16605.

69. Roca FJ, et al. TNF induces pathogenic programmed macrophage necrosis in tuberculosis through a mitochondrial-lysosomal-endoplasmic reticulum circuit. Cell. 2019;178(6):1344-1361.

70. Kim SK, et al. Palmitate induces cisternal ER expansion via the activation of XBP-1/CCT $\alpha$-mediated phospholipid accumulation in RAW 264.7 cells. Lipids Health Dis. 2015;14:73.

71. Korbecki J, Bajdak-Rusinek K. The effect of palmitic acid on inflammatory response in macrophages: an overview of molecular mechanisms. Inflamm Res. 2019;68(11):915-932.
72. Loskovich MV, et al. Inhibitory effect of palmitate on the mitochondrial NADH:ubiquinone oxidoreductase (complex I) as related to the active-de-active enzyme transition. Biochem J. 2005;387(pt 3):677-683.

73. Seifert EL, et al. Electron transport chain-dependent and -independent mechanisms of mitochondrial $\mathrm{H} 2 \mathrm{O} 2$ emission during long-chain fatty acid oxidation. J Biol Chem. 2010;285(8):5748-5758.

74. Pimenta AS, et al. Prolonged exposure to palmitate impairs fatty acid oxidation despite activation of AMP-activated protein kinase in skeletal muscle cells. J Cell Physiol. 2008;217(2):478-485.

75. Galic S, et al. Hematopoietic AMPK $\beta 1$ reduces mouse adipose tissue macrophage inflammation and insulin resistance in obesity. JClin Invest. 2011;121(12):4903-4915.

76. Riera-Borrull M, et al. Palmitate conditions macrophages for enhanced responses toward inflammatory stimuli via JNK activation. J Immunol. 2017;199(11):3858-3869.

77. Finucane OM, et al. Monounsaturated fatty acid-enriched high-fat diets impede adipose NLRP3 inflammasome-mediated IL-1 $1 \beta$ secretion and insulin resistance despite obesity. Diabetes. 2015;64(6):2116-2128.

78. Lim JH, et al. Oleic acid stimulates complete oxidation of fatty acids through protein kinase A-dependent activation of SIRT1-PGC1 $\alpha$ complex. J Biol Chem. 2013;288(10):7117-7126.

79. Agarwal P, et al. Foam cells control mycobacterium tuberculosis infection. Front Microbiol. 2020;11:1394.

80. Parihar SP, et al. Protein kinase C-delta (PKCdelta), a marker of inflammation and tuberculosis disease progression in humans, is important for optimal macrophage killing effector functions and survival in mice. Mucosal Immunol. 2018;11(2):496-511.

81. Hackett EE, et al. Mycobacterium tuberculosis limits host glycolysis and IL-1 $1 \beta$ by restriction of PFK-M via MicroRNA-21. Cell Rep. 2020;30(1):124-136.

82. Verrall AJ, et al. Early clearance of Mycobacterium tuberculosis: a new frontier in prevention. Immunology. 2014;141(4):506-513.

83. Graustein $\mathrm{AD}$, et al. Inflammasome genetic variants, macrophage function, and clinical outcomes in cystic fibrosis [published online April 13, 2021]. Am J Respir Cell Mol Biol. https://doi. org/10.1165/rcmb.2020-0257oc.

84. Horne DJ, et al. Human ULK1 variation and susceptibility to mycobacterium tuberculosis infection. J Infect Dis. 2016;214(8):1260-1267.

85. Shah JA, et al. A functional toll-interacting protein variant is associated with Bacillus Calmette-Guérin-specific immune responses and tuberculosis. Am J Respir Crit Care Med. 2017;196(4):502-511.

86. Martin M. Cutadapt removes adapter sequences from high-throughput sequencing reads. EMBnetjournal. 2011;17(1):3.

87. Dobin A, et al. STAR: ultrafast universal RNA-seq aligner. Bioinformatics. 2013;29(1):15-21.

88. Li B, Dewey CN. RSEM: accurate transcript quantification from RNA-Seq data with or without a reference genome. BMC Bioinformatics. 2011;12:323.

89. Law CW, et al. voom: Precision weights unlock linear model analysis tools for RNA-seq read counts. Genome Biol. 2014;15(2):R29.

90. Listenberger LL, et al. Palmitate-induced apoptosis can occur through a ceramide-independent pathway. J Biol Chem. 2001;276(18):14890-14895.

91. Chen $\mathrm{H}$, et al. Control for population structure and relatedness for binary traits in genetic association studies via logistic mixed models. Am J Hum Genet. 2016;98(4):653-666.

92. Gogarten SM, et al. Genetic association testing using the GENESIS R/Bioconductor package. Bioinformatics. 2019;35(24):5346-5348. 